\title{
Availability of CT on island medical facilities: Association with mortality in patients evacuated via long-distance air medical transport
}

Ayaka Ishihata ( $\square$ ayaka.ishihata@gmail.com )

Tokyo Metropolitan Hiroo Hospital

Mikio Nakajima

Tokyo Metropolitan Hiroo Hospital

Sachiko Ono

The University of Tokyo

Richard H Kaszynski

Tokyo Metropolitan Hiroo Hospital

Ryo Takane

Tokyo Metropolitan Hiroo Hospital

Masamitsu Shirokawa

Tokyo Metropolitan Hiroo Hospital

Masayuki Otawara

Tokyo Metropolitan Hiroo Hospital

Tomotsugu Nakano

Tokyo Metropolitan Hiroo Hospital

Hideaki Goto

Tokyo Metropolitan Hiroo Hospital

\section{Research Article}

Keywords: computed tomography, air medical transport, rural medicine, island, APACHE-II

Posted Date: November 9th, 2021

DOI: https://doi.org/10.21203/rs.3.rs-1036587/v1

License: (1) This work is licensed under a Creative Commons Attribution 4.0 International License. Read Full License 


\section{Abstract \\ Background}

To examine the association between availability of computed tomography (CT) and severity-of-disease/mortality in patients evacuated via long-distance emergency air medical transport from island medical facilities.

\section{Methods}

We retrospectively and consecutively enrolled patients transferred via rotary- or fixed-wing aircraft from island medical facilities to a designated emergency medical center in Tokyo, Japan between 2010 and 2020. Airlifted patients were divided into two groups, depending on the availability of CTs on the island facilities in which they were transported from. The outcomes were severity-of-disease after air medical transport and in-hospital mortality. We examined the association between these outcomes and the availability of CTs on each island facility.

\section{Results}

Eligible patients $(n=1,880)$ were categorized into the CT $(n=1,744)$ or non-CT group $(n=136)$. After adjusting for age, sex, diagnostic category and flight time, the CT group was significantly associated with greater severity of disease (coefficient, 1.30 ; $95 \%$ confidence interval, 0.13-2.47). In contrast, significant reductions in mortality were not documented in the CT group.

\section{Conclusion}

While the availability of CTs was significantly associated with greater severity of disease, CT availability did not significantly impact mortality in patients transferred from island medical facilities.

\section{Backgrounds}

Computed tomography (CT) has demonstrated its utility in delineating the presence or absence of life-threatening conditions warranting further intervention. Since their inception, CT scans have rapidly evolved to become a mainstay in emergency departments (EDs) and their numbers continue to grow globally.[1-5] Several studies have addressed the association between the availability of CTs and the frequency of emergency patient transport.[6-8] Previous observational studies have reached the general consensus that a shortage of emergency radiological services including CTs is associated with an increased frequency in patient transports from rural EDs to urban tertiary emergency healthcare facilities. While these studies offer tremendous insight into the correlation between a lack of emergency radiological services and the number of patients transports, the association between the former and patient mortality in rural environments has yet to be explored at the fine-scale level.

The present study aims to elucidate the association between CT availability and disease severity and mortality in patients evacuated via long-distance emergency air medical transport from rural island medical facilities. If the availability of CT scans has any meaningful impact on clinical outcomes in patients evacuated via long-distance emergency air medical transport, island facilities should receive these machines as part of a standard deployment package.

\section{Method}

\subsection{Setting and patients}

The Izu and Ogasawara archipelago fall under the jurisdiction of the Tokyo Metropolitan government with the former involving a string of nine islands and the latter consisting of two largely inhabited islands. Each island has a dedicated medical facility with limited medical resources depending on the size of the island population. With exception to four islands along the Izu archipelago, the majority of island medical facilities have access to CTs.[9] Imaging studies conducted via CT are transmitted to designated urban emergency medical centers for interpretation by trained radiologists and specialists. Patients identified as necessitating urgent care are airlifted via emergency air medical transport from these island facilities to a designated emergency medical center in Tokyo. With exception to 
cases that do not require CT scans for rendering a diagnosis such as post-cardiac arrest or coronary artery disease, standard protocol dictates that CT images be interpreted at a designated urban healthcare facility in Tokyo prior to making the decision to approve air medical transport. The distances from each island to Tokyo ranges from 100 to $1000 \mathrm{~km}$, and general flight times for air medical transport fall within the 0.5-5 $\mathrm{h}$ spectrum under normal circumstances.

We retrospectively enrolled consecutive patients who were transported via rotary- or fixed-wing aircraft from island medical facilities to our emergency medical center in Tokyo, Japan between April 2010 and May 2020. Patients diagnosed with post-cardiac arrest were excluded because i) these patients are transferred regardless of the results of diagnostic imaging, ii) the mortality was extremely high, and iii) the number of post-cardiac patients were relatively small. Patients with missing data were also excluded from the study. Airlifted patients were divided into two groups depending on the availability of CTs. Patients airlifted from island medical facilities with and without access to $\mathrm{CT}$ s were assigned to the $\mathrm{CT}$ and non-CT groups, respectively.

\subsection{Outcome and Variables}

The outcomes established for the present study were severity of disease and in-hospital mortality. We collected the following data for each patient: age; sex; flight time; parameters composing acute physiology and chronic health evaluation (APACHE) II score; availability of CT; and diagnosis. The APACHE-II score is a composite disease severity score widely used in the intensive care unit (ICU) setting.[10] The APACHE-II score includes 12 acute physiological scores $\left(\mathrm{AaDO}_{2}\right.$ or $\mathrm{PaO}_{2}$, body temperature, mean arterial pressure, blood $\mathrm{pH}$, heart rate, respiratory rate, serum sodium, serum potassium, creatinine, hematocrit, white blood cell count and the Glasgow Coma Scale) measured within 24 hours of admission, age points, and chronic health points. We also calculated the APACHE-II score excluding the age points and used the score as an independent variable in a multivariable logistic regression model to predict in-hospital mortality. We created this modified APACHE-II score to estimate interpretable independent effects of both age and severity in the logistic regression analysis. Diagnosis was divided into 7 categories: cerebrovascular disease, infectious disease and sepsis, gastrointestinal disease, cardiovascular disease, trauma, respiratory disease, and others. [11] Each patient had only one recorded diagnosis and these categories were mutually exclusive.

\subsection{Statistical analysis}

Continuous variables are presented as medians with interquartile ranges (IQR) and binary and categorical variables are reported as count and percentage. Differences in the variables of baseline characteristics between the non-CT and CT groups were compared using a Wilcoxon rank-sum and Chi-square tests. To examine the effectiveness of CT availability, we performed multivariable linear regression analysis with the severity assessed by APACHE-II score as a dependent variable. Independent variables included in the linear regression model were availability of $\mathrm{CT}$, age, sex, flight time, and diagnosis. Similarly, multivariable logistic regression analysis was conducted with in-hospital mortality as a dependent variable. For the logistic regression model, the APACHE-II score excluding the age points was included as an independent variable along with the same independent variables included in the linear regression model. For the effect of diagnosis on the outcomes, we presented the relative effect of each diagnosis with cerebrovascular disease as a reference standard because the diagnosis was mutually exclusive. The threshold for significance was a $p$-value less than 0.05 , and all statistical analyses were performed using Stata/MP15(Stata, Collage Station, TX, USA).

\subsection{Ethics}

This study was approved by the Institutional Review Board of Tokyo Metropolitan Hiroo Hospital (approval number: J-66). Because this study was based on secondary analyses of medical charts, the requirement for informed consent was waived.

\section{Results}

During the study period, 1930 patients were transferred and admitted to our hospital via rotary- or fixed-wing aircraft. We excluded 50 patients who met the exclusion criteria. Finally, 1880 patients were analyzed in the study (Figure 1).

Patient characteristics and crude outcomes (APACHE-II score and in-hospital mortality) are shown in Table 1. There were no significant differences observed in age, sex diagnostic categories and flight time between the non-CT and CT groups. Median age was 72 years (IQR, 59-82 years) and flight time was $70 \mathrm{~min}$ (IQR, 53-93 min). The CT group had significantly higher APACHE-II scores compared with the non-CT group [12 (IQR, 8-17) vs. 10.5 (IQR, 7-14), p=0.007]. In-hospital mortality did not significantly differ between the two groups [CT group, $8.4 \%$ vs. non-CT group, $4.4 \%, \mathrm{p}=0.10$ ]. 
Table 1

Baseline characteristics and outcomes before adjustment

\begin{tabular}{|c|c|c|c|}
\hline Variables & $\begin{array}{l}\text { Non-CT group } \\
(n=136)\end{array}$ & $\begin{array}{l}\text { CT group } \\
(n=1,744)\end{array}$ & P-value \\
\hline Age, years, median (IQR) & $68.5(51.5-83.5)$ & $72(59-82)$ & 0.25 \\
\hline Sex, male (\%) & $90(66.2)$ & $1,105(63.4)$ & 0.51 \\
\hline \multicolumn{4}{|l|}{ Diagnosis (\%) } \\
\hline Cerebrovascular disease & $21(15.4)$ & 359 (20.6) & 0.051 \\
\hline Infectious disease and sepsis & $20(14.7)$ & $261(15.0)$ & \\
\hline Gastrointestinal disease & $25(18.4)$ & $260(14.9)$ & \\
\hline Cardiovascular disease & $18(13.2)$ & $380(21.8)$ & \\
\hline Trauma & $24(17.6)$ & $243(13.9)$ & \\
\hline Respiratory disease & $16(11.8)$ & $128(7.3)$ & \\
\hline Others & $12(5.9)$ & $113(6.5)$ & \\
\hline Flight time, min, median (IQR) & $73(54-93.5)$ & $69(53-93)$ & 0.99 \\
\hline \multicolumn{4}{|l|}{ Outcomes } \\
\hline APACHE-II score, median (IQR) & $10.5(7-14)$ & $12(8-17)$ & 0.007 \\
\hline In-hospital mortality (\%) & $6(4.4)$ & $146(8.4)$ & 0.10 \\
\hline
\end{tabular}

Wilcoxon rank sum test for continuous variables and chi-square test for categorical variables were used for the comparison.

The results of multivariable linear regression analysis for disease severity assessed by APACHE-II score are shown in Table 2.

Availability of CT was significantly associated with higher APACHE-II scores excluding the age points [coefficient, 1.30; $95 \%$ confidence interval, 0.13-2.47; $p=0.029$ ]. Older age and longer flight times were also significantly associated with greater severity of disease. 
Table 2

Multivariable linear regression analysis for severity of disease assessed by APACHE-II score $(n=1,880)$

\begin{tabular}{|llll|}
\hline Variable & Coefficient & (95\% Cl) & P-value \\
\hline Availability of CT & 1.30 & $(0.13$ to 2.47$)$ & 0.029 \\
\hline Age (per year) & 0.05 & $(0.03$ to 0.07$)$ & 0.001 \\
\hline Sex, male & 0.14 & $(-0.54$ to 0.81$)$ & $<.69$ \\
\hline Flight time (per min) & -0.01 & $(-0.02$ to -0.01$)$ & $<.001$ \\
\hline Diagnosis & & & 0.36 \\
\hline Cerebrovascular disease & Reference & $(-1.64$ to 0.6$)$ & 0.005 \\
\hline Infectious disease and sepsis & -0.52 & $(-2.39$ to -0.42$)$ & 0.86 \\
\hline Gastrointestinal disease & -1.40 & $(-1.11$ to 0.92$)$ & 0.022 \\
\hline Cardiovascular disease & -0.10 & $(-2.25$ to -0.17$)$ & 0.18 \\
\hline Trauma & -1.21 & $(-0.43$ to 2.25$)$ & 0.001 \\
\hline Respiratory disease & 0.91 & $(1.28$ to 4.69$)$ & \\
\hline Others & 2.99 & & \\
\hline APACHE, acute physiology and chronic health & evaluation; Cl, confidence interval; CT, computed tomography \\
\hline
\end{tabular}

Table 3 shows the results of the multivariable logistic regression analysis for in-hospital mortality. Availability of CTs was not significantly associated with in-hospital mortality [odds ratio, 1.66; 95\% confidence interval, 0.66-4.21; $p=0.28$ ]. Older age and higher APACHE-II scores-excluding the age points-were significantly associated with higher mortality.

Table 3

Multivariable logistic regression analysis for in-hospital mortality $(n=1,880)$

\begin{tabular}{|c|c|c|c|}
\hline Variables & Odds ratio & $(95 \% \mathrm{Cl})$ & P-value \\
\hline Availability of CT & 1.66 & (0.66 to 4.21$)$ & 0.28 \\
\hline Age (per year) & 1.03 & (1.01 to 1.04$)$ & $<0.001$ \\
\hline Sex, male & 0.99 & (0.67 to 1.48$)$ & 0.97 \\
\hline Flight time (per min) & 1.00 & (0.99 to 1.00$)$ & 0.051 \\
\hline \multicolumn{4}{|l|}{ Diagnosis } \\
\hline Cerebrovascular disease & \multicolumn{3}{|l|}{ Reference } \\
\hline Infectious disease and sepsis & 0.77 & (0.40 to 1.46$)$ & 0.42 \\
\hline Gastrointestinal disease & 0.79 & (0.41 to 1.52$)$ & 0.48 \\
\hline Cardiovascular disease & 0.83 & (0.48 to 1.45$)$ & 0.51 \\
\hline Trauma & 0.94 & (0.50 to 1.77$)$ & 0.85 \\
\hline Respiratory disease & 2.09 & (1.08 to 4.06$)$ & 0.029 \\
\hline Others & 0.58 & (0.25 to 1.34$)$ & 0.20 \\
\hline APACHE-II score ${ }^{\dagger}$ & 1.14 & (1.12 to 1.17$)$ & $<0.001$ \\
\hline \multicolumn{4}{|c|}{$\mathrm{Cl}$, confidence interval; $\mathrm{CT}$, computed tomography; $\mathrm{APACHE}$, acute physiology and chronic health evaluation } \\
\hline
\end{tabular}

\section{Discussion}


This study aimed to assess the relationship between the availability of CTs on island medical facilities and severity-of-disease and inhospital mortality in patients transferred to a designated tertiary medical facility via rotary-or fixed-wing aircraft. The availability of CTs was associated with higher APACHE-II scores but not in-hospital mortality.

Previous observational studies have shown that the availability of CTs and other radiological services reduced the number of patient transfers. [6-8] Consistent with these findings, the present study identified a significant association between the availability of CTs and greater disease severity. Therefore, the availability of CTs may have enabled medical facilities to avoid unnecessary patient transfers. In addition to examining the association of CT availability with disease severity, the present study also endeavored to examine any association with mortality; however, no significant association was observed in relation to CT availability and this outcome. The underlying narrative that can be inferred from these results is relatively clear. The lack of CTs on island medical facilities does not necessarily result in worse outcomes given an appropriate transportation infrastructure is in place.

On the opposite end of the spectrum, the lack of CTs results in over-triage and ultimately unwarranted emergency air medical transports. In general, the cost burden of aerial transport is immensely high. At the core of these discussions lies the central debate on permanent CT placement versus incidental emergency air transport--which is more cost effective? The answer to this question could not be addressed in the present study and therefore a comparison involving the medico-economical implications should be considered in a future study in order to dissect the cost benefit correlations of incidental emergency air medical transport at the fine scale level.

There are several limitations which should be noted in the present study. Only patients transported to a single medical institution were included in the present study, which could impact the generalizability of its findings. It should, however, be noted that the single facility examined in this study accounts for approximately $90 \%$ of all emergency air medical transportation cases in Tokyo. Second, as a de facto practice, island medical facilities with only one stationed physician are not granted permanent placement of CT scanners. As such there are inter-island discrepancies with regard to human and infrastructural resources. Third, the APACHE-II score in the present study was measured within 24 hours of admission at the receiving urban healthcare facility and does not reflect any changes in severity occurring during air medical transport. In addition to the above limitations, the present study only examined the association between CT availability and clinical outcome. It is important to note that the availability of CTs does not imply its utilization. Therefore it remains unknown whether CT assessments were performed on each of the cases included in this study. These factors could have in advertently biased the results of the present analysis.

\section{Conclusion}

While the availability of CTs was significantly associated with severity of disease, CT availability did not significantly impact the outcome of mortality in patients transferred from island medical facilities. The present study suggests that the availability of CTs could possibly decrease the number of unwarranted emergency air medical transports from island medical facilities.

\section{Abbreviations}

$\mathrm{CT}$, computed tomography; ED, emergency department; APACHE, acute physiology and chronic health evaluation; IQR, interquartile range

\section{Declarations}

Ethics approval and consent to participate; This study was approved by the Institutional Review Board of Tokyo Metropolitan Hiroo Hospital (approval number: J-66). Because this study was based on secondary analyses of medical charts, the requirement for informed consent was waived.

Consent for publication; Not applicable

Availability of data and materials; Not applicable

Competing interests; The authors declare that they have no competing interest

Funding ; Not applicable

Authors' contributions

Page $6 / 8$ 
Al, Conceptualization, Methodology, Software and Writing - Original draft; MN, Conceptualization, Methodology, Software, Data Curation and Writing - Review \& Editing; SO, Statistical Processing RHK, Writing -Review \& Editing; RT, Resources; MS, Resources; MO,

Supervision; TN, Supervision; HG, Supervision

\section{Acknowledgements}

We are indebted to staff members who treated patients in each island clinic and introduced patients to our facility; and to the Tokyo Fire Department and Maritime Self-Defense Force who collaborated with us on the air medical transport.

\section{References}

1. Larson DB, Johnson LW, Schnell BM, Salisbury SR, Forman HP. National trends in CT use in the emergency department: 1995-2007. Radiology. 2011;258:164-73.

2. Kocher KE, Meurer WJ, Fazel R, Scott PA, Krumholz HM, Nallamothu BK. National trends in use of computed tomography in the emergency department. Ann Emerg Med. 2011;58:452-62.e3. http://dx.doi.org/10.1016/j.annemergmed.2011.05.020

3. Hu SY, Hsieh MS, Lin MY, et al. Trends of CT utilisation in an emergency department in Taiwan: A 5-year retrospective study. BMJ Open. 2016;6:e010973.

4. Oh HY, Kim EY, Cho J, et al. Trends of CT use in the adult emergency department in a tertiary academic hospital of Korea during 2001-2010. Korean J Radiol. 2012;13:536-40.

5. Zhou JC, Zheng SW, Yu YX, et al. Trends in computed tomography utilization and association with hospital outcomes in a chinese emergency department. PLoS One. 2012;7:1-6.

6. Bergeron C, Fleet R, Tounkara FK, Lavallée-Bourget I, Turgeon-Pelchat C. Lack of CT scanner in a rural emergency department increases inter-facility transfers: A pilot study. BMC Res Notes. 2017;10:1-6. https://doi.org/10.1186/s13104-017-3071-1

7. Lyon M, Sturgis L, Lendermon D, et al. Rural ED transfers due to lack of radiology services. Am J Emerg Med. 2015;33:1630-4.

8. Rourke JTB, Kennard MA. Emergency patient transfers from rural hospitals: A regional study. Can J Emerg Med. 2001;3:296-301.

9. Tokyo Metropolitan Government. Medical care resources in Izu and Ogasawara Islands. 2018. https://www.fukushihoken.metro.tokyo.lg.jp/iryo/iryo_hoken/ritousankan/hekiti_kyogikai/R2_kyogikai.files/28_iryokaigoshigen.pdf (Article in Japanese) [Accessed 28 October, 2021].

10. Knaus WA, Draper EA, Wagner DP, Zimmerman JE. APACHE II: a severity of disease classification system. Crit Care Med. 1985;13:818-29.

11. Nakajima M, Aso S, Yasunaga H, Shirokawa M, Nakano T, Miyakuni Y, et al. Body temperature change and outcomes in patients undergoing long-distance air medical transport. Am J Emerg Med. 2019;37:89-93. https://doi.org/10.1016/j.ajem.2018.04.064

\section{Figures}


Patients evacuated from island medical facilities via emergency air medical transport

from April 2010 to May $2020(n=1,930)$

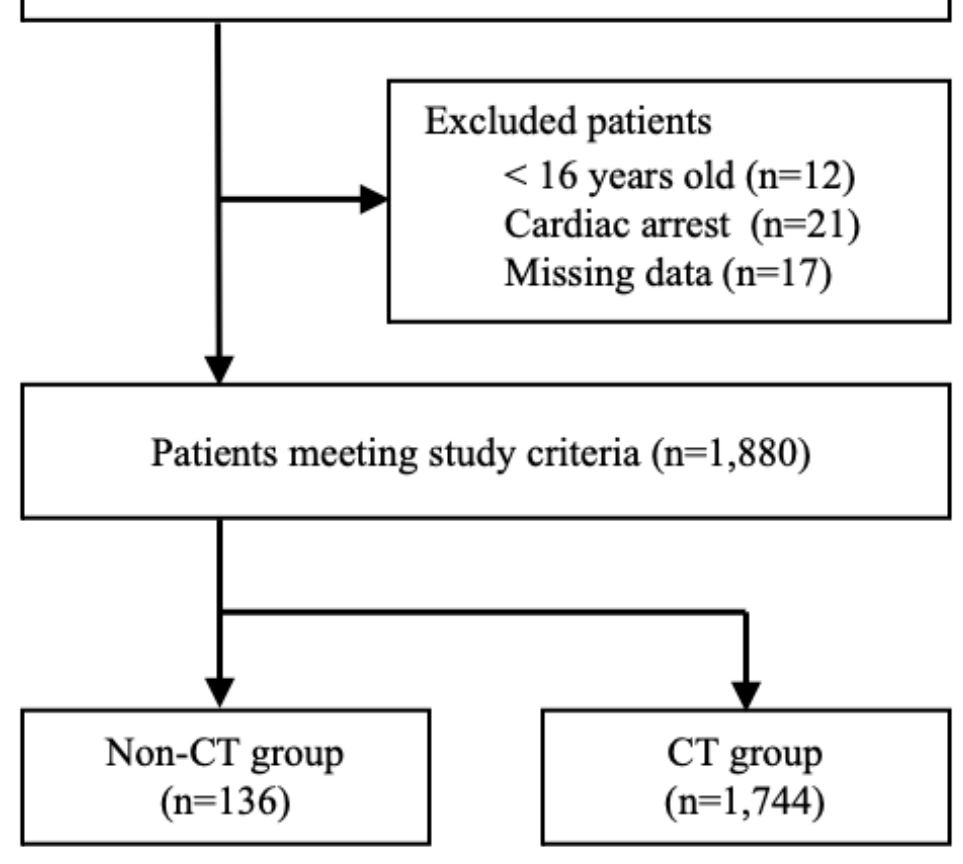

\section{Figure 1}

Flow diagram for selecting the study population CT, computed tomography 